\title{
ONLINE IDENTIFICATION OF THE ELECTRICALLY STIMULATED QUADRICEPS MUSCLE GROUP
}

\author{
T. Schauer ${ }^{*}$ F. Previdi ${ }^{\dagger}$ K. J. Hunt ${ }^{\ddagger}$ E. Ferchland ${ }^{*}$ N.-O. Negård ${ }^{*}$ \\ J. Raisch * \\ * Max Planck Institute for Dynamics of Complex Technical Systems, \\ Sandtorstr. 1, D-39106 Magdeburg, Germany \\ E-mail: schauer@mpi-magdeburg.mpg.de \\ ${ }^{\dagger}$ Dipartimento di Ingegneria, Universitá di Bergamo, \\ via Marconi 5, 24044 Dalmine (BG), Italy \\ $\ddagger$ Centre for Rehabilitation Engineering, Dept. of Mechanical Eng., \\ University of Glasgow, Glasgow GI2 8QQ, Scotland
}

\begin{abstract}
In this paper, a new approach for estimating a nonlinear model of the electrically stimulated quadriceps muscle group under non-isometric conditions is investigated. In order to identify the muscle dynamics (stimulation pulse width-active knee moment relation) from discrete-time angle measurements only, a hybrid model structure is postulated for the shankquadriceps dynamics. The model consists of a relatively well known time-invariant passive component and an uncertain time-variant active component. Rigid body dynamics, described by the Equation of Motion (EoM), and passive joint properties form the time-invariant part. The actuator, i.e. the electrically stimulated muscle group, represents the uncertain time-varying section. A recursive algorithm is outlined for identifying online the stimulated quadriceps muscle group. The algorithm requires EoM and passive joint characteristics to be known a priori. The muscle dynamics represent the product of a continuous-time nonlinear activation dynamics and a nonlinear static contraction function described by a Normalised Radial Basis Function (NRBF) network which has knee-joint angle and angular velocity as input arguments. An Extended Kalman Filter (EKF) approach is chosen to estimate muscle dynamics parameters and to obtain full state estimates of the shank-quadriceps dynamics simultaneously. Copyright (C) 2003 IFAC
\end{abstract}

Keywords: Electrical Stimulation, Extended Kalman Filters, Physiological Models, Neural Networks

\section{INTRODUCTION}

The development of controlled neuroprostheses for the restoration of basic motor functions to paraplegics represents a major research area in rehabilitation engineering. These lower limb prostheses are based on a technique called Functional Electrical Stimulation (FES), in which stimulation of paralysed muscles can generate muscle contraction (Popović and Sinkjær, 2000). FES, together with appropriate sensor and control technology, can provide useful, controlled functional movement. To design controllers for FES systems, it can be useful to have an accurate model which describes the relation between the stimulation parameters and the resulting force, moment or movement.

In this paper we are particularly interested in a nonlinear modelling approach for the electrically stimulated quadriceps muscle group under non-isometric conditions.

When modelling these dynamics, the combination of a modified Hill-type muscle model and a passive model part is often assumed. The latter consists of the Equa- 
tion of Motion (EoM), passive elastic and passive viscous properties; first principles, simple pendulum tests and static force measurements without stimulation are used to obtain this model part.

Detailed muscle models describe submuscle groups of the quadriceps muscle group separately. Assuming a standard Hill-type muscle model, the generated muscle force of the individual muscle groups is the product of muscle activation (described by a nonlinear recruitment curve and a linear dynamics with time delay), a nonlinear force - muscle length relation and a nonlinear force - muscle contraction velocity relation. To calculate the resulting knee moment, nonlinear moment arms (parameterised by the joint angle) for the muscles with respect to the knee-joint have to be known. Passive muscle properties are usually assigned to the joint. The identification of such muscle models is not possible or too complicated in vivo, so that parameters are often taken from the literature that leads to a bad fit of the models to individual subjects.

Alternatively, the quadriceps can be treated as a single muscle, and joint moment generation can be directly described in the joint angle - angular velocity space instead of the muscle length - muscle contraction velocity space. To keep the identification task simple, often linear moment - joint angle and moment - angular velocity relations are postulated, which are only valid in a limited subrange of the entire joint angle - velocity range.

Identification of the quadriceps dynamics (stimulation - active knee moment relation) from shank movements was investigated in (Chizeck et al., 1999) (online estimation) and in (Franken et al., 1995; Ferrarin and Pedotti, 2000) (off-line estimation). All these approaches require, in addition to angle information, either direct measurements of velocity and acceleration or their numerical computation from frequently sampled kneejoint angle measurements.

In this paper, we present a simple approach to estimate the nonlinear muscle model from knee-joint angle measurements only, sampled with stimulation frequency. Further, we look for a general nonlinear function that describes the active knee moment as a function of joint angle and angular velocity.

\section{EXPERIMENTAL SETUP}

A 57 year-old T10 complete paraplegic male subject, 2 years post-injury, training his quadriceps muscles for an FES-cycling project, participated in this study.

The experimental set-up is depicted in Fig. 1. The subject is seated on a bed with the unloaded shank free to swing. Pulsewidth modulated electrical current pulses are sent to the knee extensor muscles via surface electrodes and cause contractions and knee-joint motion. The knee angle $x_{1}$ (system output) is measured and sampled every $50 \mathrm{~ms}$ by a $3 \mathrm{D}$-motion anal-

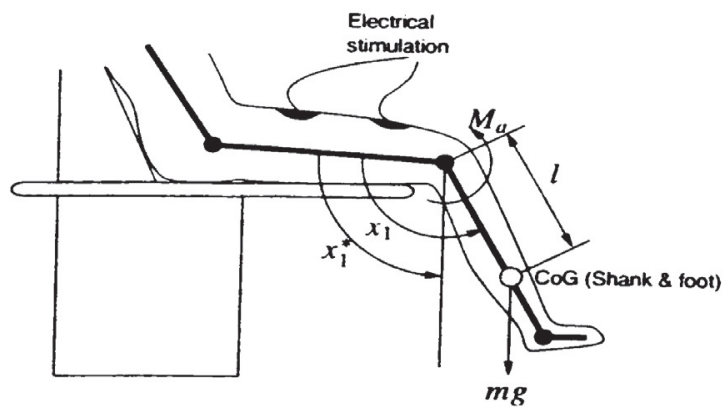

Fig. 1. Experimentel set-up.

ysis system working with ultrasound. It delivers the angle measurements in realtime via the parallel port to a laptop. A multi-channel stimulator is connected to the laptop via the serial port and delivers the current pulses with pulsewidth $p w$ up to $800 \mu \mathrm{s}$. During the experiments the pulsewidth serves as a variable input signal whereas the current amplitude $I=80 \mathrm{~mA}$ and the stimulation frequency $f=20 \mathrm{~Hz}$ are fixed.

The input signal during identification is a Multi-level Pseudo-Random Sequence (M-level PRS) (Godfrey, 1993) in order to excite the nonlinear system behaviour. An M-level PRS is a periodic, deterministic signal which has an autocorrelation function similar to white noise.

\section{METHODS}

\subsection{Model Structure}

The shank-quadriceps dynamics are modelled as the interconnection of a passive part, including equation of motion and passive elastic/viscous joint properties, and an active part, consisting of muscle activation and contraction dynamics. Passive muscle properties have been assigned to the joint in order to keep the number of muscle parameters small. The passive system part is nearly time-invariant, whereas the active part (muscle actuator) possesses a large day-to-day variation and is affected by muscle fatigue due to prolonged stimulation.

Passive Viscous-Elastic Characteristics and Equation of Motion. The total knee-joint moment is given by

$$
M_{K}=M_{e}+M_{g}+M_{v}+M_{a}
$$

with $M_{e}$ : passive elastic moment, $M_{g}$ : gravitational moment, $\boldsymbol{M}_{v}$ : passive viscous moment and $\boldsymbol{M}_{\boldsymbol{a}}$ : active moment generated by quadriceps stimulation. The gravitational component is determined by

$$
M_{g}\left(x_{1}\right)=-m g l \sin \left(x_{1}-x_{1}^{*}\right)
$$

where $m$ is the mass of the shank-foot complex and $l$ is the distance between the knee and centre of mass. The knee-joint angle $x_{1}$ as well as the angle $x_{1}^{*}$ are defined in Fig. 1. The elastic moment $M_{e}\left(x_{1}\right)$ is expressed as 


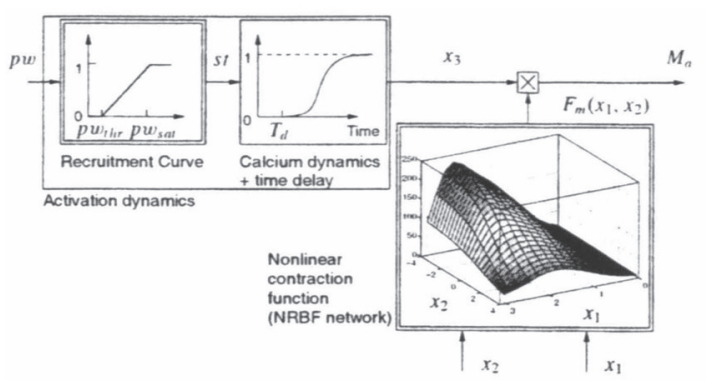

Fig. 2. Model of the quadriceps muscle group

an exponential function as proposed and experimentally verified in (Ferrarin and Pedotti, 2000). Passive viscous joint properties have been modelled as nonlinear damping function

$$
M_{v}\left(x_{2}\right)=B_{1} \tanh \left(-B_{2} x_{2}\right)-B_{3} x_{2},
$$

where $B_{1}, B_{2}$ and $B_{3}$ are positive constants and $x_{2}$ the angular velocity. Note that Coulombic friction effects are captured by this damping function. The Equation of Motion (EoM) is given by

$$
\dot{x}_{2}=\frac{1}{J_{K}} M_{K}\left(x_{1}, x_{2}, M_{a}\right)
$$

where $J_{K}$ represents the moment of inertia of the shank-foot complex about the knee-joint. The anthropometric parameters $m, J_{K}$ and $l$ have been estimated by measuring the height and weight of the subject and using regression equations. A passive pendulum trial was performed to obtain stiffness and damping parameters.

Nonlinear Muscle Model The active muscle moment $M_{a}$ is determined by the product of an activation dynamics and a nonlinear static contraction function (cf. Fig. 2).

The activation dynamics consist of a static recruitment curve and a transfer function connected in series. The recruitment curve gives a measurement for the normalised amount of activated motor units depending on the pulsewidth $p w$. This measurement is labelled as stimulation level. A piecewise linear function describes the recruitment curve:

$$
s t=\left\{\begin{array}{cl}
0 & , p w<p w_{t h r} \\
\frac{p w-p w_{t h r}}{p w_{s a t}-p w_{t h r}}, & p w_{t h r} \leq p w \leq p w_{s a t} \\
1 & , p w>p w_{s a t}
\end{array}\right.
$$

where $p w_{t h r}$ is the pulsewidth for which first motor units are recruited and $p w_{\text {sat }}$ stands for the pulsewidth where all motor units are recruited. These threshold and saturation levels can be determined as follows under non-isometric conditions: the pulsewidth for which the shank starts to move is taken as $p w_{t h r}$, and the pulsewidth for which the leg reaches full extension is approximately chosen as $p w_{\text {sat }}$.

The temporal behaviour of the muscle activation is mainly influenced by calcium $\mathrm{Ca}^{2+}$ release dynamics, finite conduction velocities in the membrane system and delays from the chemical reactions involved. To describe these effects a second order transfer function with an input time delay $T_{d}$ is used:

$$
\begin{aligned}
& \dot{x}_{3}=x_{4} \\
& \dot{x}_{4}=-2 w_{0} D x_{4}-w_{0}^{2} x_{3}+w_{0}^{2} s t\left(t-T_{d}\right)
\end{aligned}
$$

where the output $x_{3}$ is the normalised activation of the muscle, $w_{0}$ and $D=1$ are the undamped natural frequency and the damping factor of the calcium dynamics respectively.

Nonlinear Contraction Function. The active knee moment produced by the quadriceps is given by

$$
M_{a}=F_{m}\left(x_{p}\right) x_{3}
$$

where $F_{m}[\mathrm{Nm}]$ is the nonlinear contraction function which defines the maximal possible active torque in the case of full muscle activation dependent on kneejoint angle and velocity which have been arranged in a vector $x_{p}=\left[\begin{array}{ll}x_{1} & x_{2}\end{array}\right]^{\prime}$.

With the aim to have little constraints on the form of the nonlinear contraction function, a Normalised Radial Basis Function (NRBF) network (Nelles, 2001) is used to parameterise $F_{m}$ :

$$
F_{m}\left(x_{p}\right)=\sum_{i=1}^{M} \psi_{i} \tilde{\varphi}_{i}\left(\left\|x_{p}-c_{i}\right\| \Sigma_{i}\right)
$$

Here, the contraction function is described as the sum of $M$ normalised radial basis functions $\tilde{\varphi}_{i}, i=$ $1, \ldots, M$ which are weighted by the constants $\psi_{i}, i=$ $1, \ldots, M$. The function $\tilde{\varphi}_{l}$ is given by

$$
\bar{\varphi}_{i}\left(\left\|x_{p}-c_{i}\right\| \Sigma_{l}\right)=\frac{\varphi_{l}\left(\left\|x_{p}-c_{i}\right\| \Sigma_{l}\right)}{\sum_{l=1}^{M} \varphi_{l}\left(\left\|x_{p}-c_{l}\right\| \Sigma_{l}\right)}
$$

where $\varphi_{i}$ is a non-normalised Radial Basis Function (RBF) chosen to be a Gaussian function:

$$
\varphi_{i}\left(\left\|x_{p}-c_{i}\right\| \Sigma_{i}\right)=\exp \left(-1 / 2\left\|x_{p}-c_{i}\right\|_{\Sigma_{i}}^{2}\right) .
$$

The argument $\left\|\boldsymbol{x}_{p}-\boldsymbol{c}_{i}\right\|_{\Sigma_{t}}$ represents the distance of the NRBF network input vector $\boldsymbol{x}_{p}$ from the centre vector $c_{i}$ and is calculated by

$$
\left\|x_{p}-c_{i}\right\|_{\Sigma_{i}}=\sqrt{\left(x_{p}-c_{i}\right)^{\prime} \Sigma_{i}\left(x_{p}-c_{i}\right)}
$$

with $\Sigma_{i}$ being a norm matrix. For this application the $\Sigma_{i}$ matrix is chosen to be diagonal; thus, it contains the inverse variances for each input dimension.

State-Space Model Representation. The shank-quadriceps dynamics can be compactly written as a statespace model with the following state vector $x$, output $y$ and input $u$ 


$$
\begin{aligned}
& x=\left[\begin{array}{llll}
x_{1} & x_{2} & x_{3} & x_{4}
\end{array}\right]^{\prime} \\
& y=x_{1} \\
& u=p w\left(t-T_{d}\right) .
\end{aligned}
$$

Elements of the state vector $\boldsymbol{x}$ are joint angle and velocity as well as the states of the calcium dynamics. The knee-joint angle is defined as output, and the time delayed pulsewidth is taken as system input. The form of the state-space model is then

$$
\begin{aligned}
& \dot{x}=f(x, u) \\
& y=c^{\prime} x
\end{aligned}
$$

with the vector function $\boldsymbol{f}$ given by

$$
\begin{aligned}
& f(x, u)= \\
& {\left[\begin{array}{c}
x_{2} \\
\frac{1}{J_{K}}\left[M_{e}\left(x_{1}\right)+M_{g}\left(x_{1}\right)+M_{v}\left(x_{2}\right)+F_{m}\left(x_{p}\right) x_{3}\right] \\
x_{4} \\
-2 w_{0} D x_{4}-w_{0}^{2} x_{3}+w_{0}^{2} s t(u)
\end{array}\right]}
\end{aligned}
$$

The vector $c$ is defined as $c=\left[\begin{array}{llll}1 & 0 & 0 & 0\end{array}\right]^{\prime}$.

\subsection{Online Estimation of System States and Muscle Parameters}

Online estimation of the state vector $\boldsymbol{x}$ and some muscle parameters is investigated. Muscle parameters, considered for online estimation, are the weights of the NRBF network which are arranged in a vector $\psi=\left[\psi_{1}, \cdots, \psi_{M}\right]^{\prime}$, as well as the frequency $w_{0}$ of the activation dynamics.

All parameters to be tracked form a parameter vector $\boldsymbol{\theta}:$

$$
\boldsymbol{\theta}=\left[\begin{array}{ll}
w_{0} & \boldsymbol{\psi}^{\prime}
\end{array}\right]^{\prime} .
$$

A common approach in parameter estimation is to model the parameters that have to be estimated by a differential equation. By setting

$$
\dot{\boldsymbol{\theta}}=\mathbf{0}
$$

we assume that model parameters are time-invariant or slowly varying in comparison to the process dynamics. Equation (4) is added to the plant state-space model and a nonlinear observer is then applied to estimate the meta-state $z=\left[\begin{array}{ll}x^{\prime} & \theta^{\prime}\end{array}\right]^{\prime}$. The resulting dynamical system for the meta-state is given by

$$
\begin{gathered}
\dot{z}=\left[\begin{array}{c}
\dot{x} \\
\dot{\theta}
\end{array}\right]=\underbrace{\left[\begin{array}{c}
f(x, u, \theta) \\
0
\end{array}\right]}_{f^{\prime}(z, u)} \\
y=\underbrace{\left[\begin{array}{ll}
c^{\prime} & 0
\end{array}\right]}_{c^{z^{\prime}}}\left[\begin{array}{l}
x \\
\theta
\end{array}\right] .
\end{gathered}
$$

The estimation of the meta-state is carried out using an Extended Kalman Filter (EKF) under following conditions and assumptions:
- Only discrete-time noisy angle measurements are available as observations (sample time $T_{s}=50 \mathrm{~ms}$ ).

- The input signal is constant between two pulses: $u(t)=u_{k}=u\left(k T_{s}\right), \quad k T_{s} \leq t<(k+1) T_{s}$.

- Estimates $\hat{z}_{k}=\hat{z}\left(k T_{s}\right)$ of the meta-state are required at discrete-time instants only.

To perform this estimation task, we assume that a discrete-time state-space model of the shank-quadriceps dynamics is available, that describes the system (5-6) exactly at the time instants $k T_{s}$. The discretetime model has the following form:

$$
\begin{aligned}
z_{k} & =f_{s}^{z}\left(z_{k-1}, u_{k-1}\right)+v_{k-1} \\
y(k) & =c^{z^{\prime}} z_{k}+r_{k}
\end{aligned}
$$

where $f_{s}^{z}$ is an appropriate transition function. Measurement noise $r_{k}$ and process noise $v_{k}$ are uncorrelated sampled white noise signals with covariance $\mathcal{E}\left(r_{n} r_{k}\right)=\delta_{n, k} R_{k}$ and covariance matrix $\varepsilon\left(v_{n} v_{k}^{\prime}\right)=$ $\delta_{n, k} \boldsymbol{V}_{k}$ respectively. Here, $\mathcal{E}$ is the expectation operator and $\delta$ is the Kronecker symbol.

The EKF provides an efficient method for generating approximate maximum-likelihood estimates of the meta-state of the discrete-time system (7-8). The filter optimally combines noisy observations with predictions from the known dynamical model and involves the following recursive procedure (Friedland, 1996):

\section{Ist step: Propagate the state}

$$
\tilde{z}_{k}=f_{s}^{z}\left(\hat{z}_{k-1}, u_{k-1}\right)
$$

Here, $\bar{z}_{k}$ is the a priori state estimate, and represents the state estimate immediately before the $k$ th observation of the knee-joint angle. This estimate is based on the estimate $\hat{z}_{k-1}$ which is the state estimate after the $(k-1)$ th observation of the previous time step. The state estimate immediately after an observation is called the a posteriori estimate.

As the transition matrix $\boldsymbol{f}_{s}^{z}$ is not known explicitly, it will be defined implicitly as the solution of the nonlinear differential equation (5). The estimate $\tilde{z}_{k}$ is the result of numerical integration of (5) over the interval $(k-1) T_{s} \leq t \leq k T_{s}$ (during which the control signal $u(t)=u_{k-1}$ is constant) starting with the initial condition $z\left((k-1) T_{s}\right)=\hat{z}_{k-1}$.

\section{2nd step: Propagate the error covariance matrix} The a priori (before observation) estimation error covariance matrix $\overline{\boldsymbol{P}}_{\boldsymbol{k}}$ is updated by

$$
\tilde{\boldsymbol{P}}_{k}=\boldsymbol{\Phi}_{k-1} \hat{\boldsymbol{P}}_{k-1} \boldsymbol{\Phi}_{k-1}^{\prime}+\boldsymbol{V}_{k}
$$

where the matrix $\boldsymbol{\Phi}_{k-1}$ is the Jacobian matrix of the transition function $f_{s}^{z}$ : 


$$
\boldsymbol{\Phi}_{k-1}=\left.\frac{\partial \boldsymbol{f}_{s}^{z}\left(z_{k-1}, u_{k-1}\right)}{\partial z_{k-1}}\right|_{z_{k-1}=\hat{z}_{k-1}} .
$$

As the transition function $f_{s}^{z}$ is not explicitly given, the matrix $\boldsymbol{\Phi}_{k-1}$ is calculated by numerical integration of the linearised matrix equation

$$
\dot{\Phi}=\boldsymbol{A}(t) \boldsymbol{\Phi}
$$

over the interval $(k-1) T_{s} \leq t \leq k T_{s}$ starting with initial conditions $\Phi\left((k-1) T_{s}\right)=I$ and with $\boldsymbol{A}(t)$ being the Jacobian matrix of the nonlinear function $f^{z}\left(z, u_{k-1}\right)$ :

$$
\boldsymbol{A}(t)=\left.\frac{\partial \boldsymbol{f}^{z}\left(z, u_{k-1}\right)}{\partial z}\right|_{z(t)}
$$

The meaning of the subscript $z(t)$ is that the partial derivatives are computed along the trajectories in state-space defined by numerical integration of the continuous-time process equation (5).

\section{3rd step: Update the Kalman gain}

The current Kalman gain is computed from the $a$ priori error covariance matrix $\tilde{\boldsymbol{P}}_{k}$ as follows:

$$
\boldsymbol{K}_{k}=\tilde{\boldsymbol{P}}_{k} \boldsymbol{c}^{z}\left(\boldsymbol{c}^{z^{\prime}} \tilde{\boldsymbol{P}}_{k} \boldsymbol{c}^{z}+R_{k}\right)^{-1}
$$

\section{4th step: State estimation update}

The $a$ priori state estimate is corrected by

$$
\hat{z}_{k}=\tilde{z}_{k}+K_{k}\left(y_{k}-c^{\prime} \tilde{z}_{k}\right)
$$

using the computed Kalman gain and the current observation $y_{k}$ in order to obtain the a posteriori state estimate $\hat{z}(k)$.

\section{5th step: Update the error covariance}

Finally, the a posteriori error covariance is given by

$$
\hat{\boldsymbol{P}}_{k}=\left(\boldsymbol{I}-\boldsymbol{K}_{k} \boldsymbol{c}^{z^{\prime}}\right) \tilde{\boldsymbol{P}}_{k}
$$

Note that the terms a priori and a posteriori for the estimation error covariance matrix depend only on the observation times and not on the observation data.

The initial approximate error covariance matrix $\hat{P}(0)$ for the EKF is chosen to be a diagonal matrix; the scalar diagonal elements of $\hat{P}(0)$ have the following values: 10 if related to plant state estimates, 1000 if related to parameter estimates of recruitment curve and calcium dynamics and 10000 if related to NRBF network weights. Larger values indicate that there is a larger discrepancy between initialisation and real values.

Low level state noise is assumed for the states which represent parameters. This prevents the gain matrix of the EKF from becoming too small. As a consequence, changes in the muscle parameters can be quickly detected also when the Kalman filter is running for a long time. The noise related to the states $x_{2}$ and $x_{4}$ is modelled with higher variance since large unpredictable variations (disturbances) in the neural activation and the knee moment are likely. The noise covariance $R_{k}$ is set according to the specifications of the measurement system.

\section{RESULTS AND DISCUSSION}

The EKF approach was evaluated in experiments with one paraplegic male subject. Fig. 3 shows an applied stimulation sequence and the corresponding measured output signal (angle) as well as the a priori angle estimate of the EKF. The corresponding parameter estimates of the muscle dynamics are depicted in Fig. 4: the upper graph shows the parameter $\hat{w}_{0}$ of the activation dynamics while elements of the estimated vector $\hat{\psi}$ are shown in the lower graph. The estimated nonlinear contraction function $\hat{F}_{m}$ after $80 \mathrm{~s}$ identification is shown in Fig. 5.

To describe the function $F_{m}$, nine RBFs $(M=9)$ were used whereas centres of the Gaussian functions are chosen as the points of a regular grid; these points cover the interesting angle-velocity region during the non-isometric muscles contractions. Variances of all non-normalised RBFs are the same. Significant overlapping of the non-normalised RBFs was selected to guarantee a high smoothness of the resulting nonlinear contraction function $F_{m}$. The elements of the metastate vector were initialised as follows:

$$
\hat{x}_{1}(0)=y(0), \hat{x}_{2}(0)=0, \hat{x}_{3}(0)=0 \text {, and } \hat{x}_{4}(0)=0 \text {. }
$$

The nonlinear recruitment curve was experimentally determined as described in Sec. 3.1. The initial estimate for the frequency $w_{0}$ was taken from the literature. The time delay $T_{d}$ was estimated by correlation analysis of recorded input-output data of the system. Initial estimates $\hat{\psi}_{i}(0), i=1, \ldots, M$, for the NRBF network weights were randomly chosen from the range $[-0.1,0.1]$.

The estimated model after $80 \mathrm{~s}$ identification was further used to simulate the plant behaviour for the time range $80-105 \mathrm{~s}$. For this test, the model is only driven by the input signal while online muscle identification and state estimation are switched off. The result of this simulation test is given in Fig. 6.

The EKF converges steadily. The one step ahead angle prediction of the EKF (a priori estimate) of the EKF fits the measured angle very well. However, the simulation test shows some discrepancies between model output and measured angle. This may be the effect of choosing a model structure which is too simple. Note that no enhanced optimisation of the NRBF network structure (no. of NRBF etc.) was carried out. 

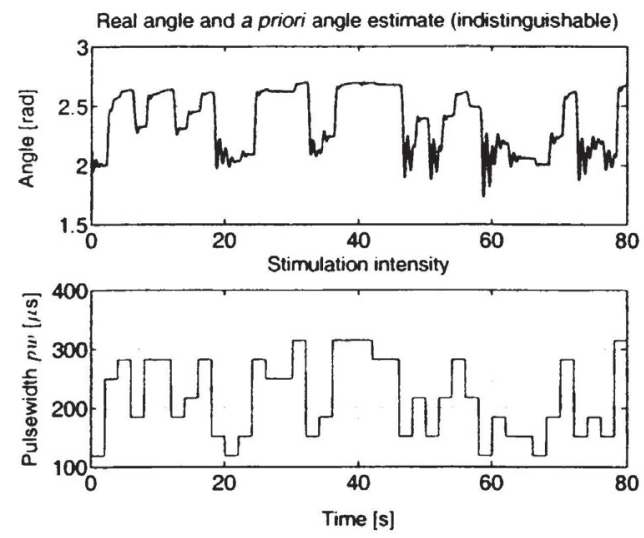

Fig. 3. 1/O data set and a priori angle estimate.
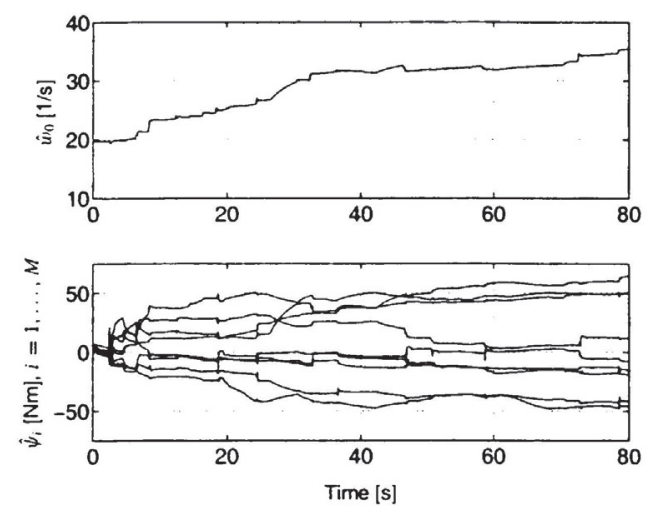

Fig. 4. Muscle parameter estimates.

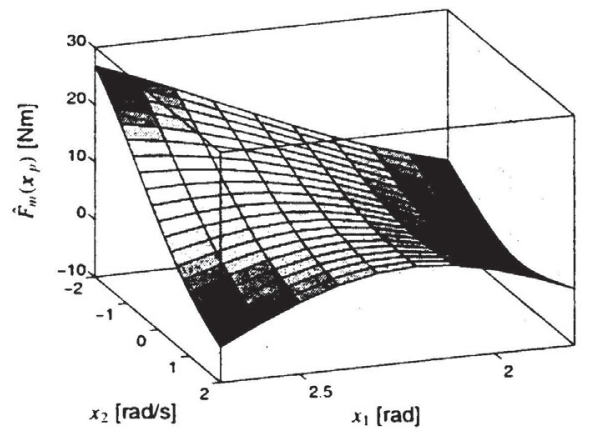

Fig. 5. Estimate of the contraction function $\hat{F}_{m}\left(\boldsymbol{x}_{p}\right)$.

In the experiments carried out for this work, mainly the vasti muscles were stimulated. In this way, the contraction function can be explained using a single classical Hill type model. The additional stimulation of the rectus femoris muscle would have probably lead to a contraction function not describable using a single classical Hill type model. However, notice that the proposed method to estimate the contraction function is able to cope with more complex situations, including the simultaneous stimulation of different muscles.
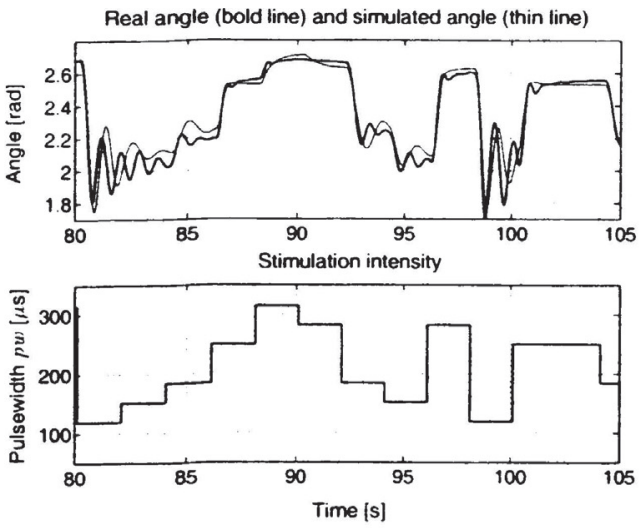

Fig. 6. Simulation test.

\section{CONCLUSIONS}

The quadriceps dynamics can be described by the proposed model structure (modified Hill-type model). An Extended Kalman Filter represents a good tool to estimate the model parameters and gives state estimates of the quadriceps-shank dynamic at the same time. A good convergence of the estimation could be shown in real experiments.

As only discrete-time joint angle measurements are required for the parameter and state estimation this method is appealing for use in a clinical set-up; it is simple and no expensive sensors for velocity and acceleration measurements are required. However, the algorithm is computational expensive, but computational power of standard processors is growing steadily, and the real-time implementation of the algorithm is feasible. The integration of the EKF in nonlinear adaptive strategies for control of the paralysed lower limbs by means of FES is planned.

\section{REFERENCES}

Chizeck, H. J., S. Chang, R. B. Stein, A. Scheiner and D. C. Ferencz (1999). Identification of electrically stimulated quadriceps muscles in paraplegic subjects. IEEE Trans. Biomed. Eng. 46(1), 51.

Ferrarin, M. and A. Pedotti (2000). The relationship between. electrical stimulus and joint torque: A dynamic model. IEEE Trans. Rehabil. Eng. 8(3), 342-352.

Franken, H. M., P. H. Veltink, R. Tijsmans, H. Nijmeier and H. B. K. Boom (1995). Identification of Quadriceps-Shank Dynamics Using Randomized Interpulse Interval Stimulation. IEEE Trans. Rehabil. Eng. 3(2), 182-190.

Friedland, B. (1996). Advanced Control System Design. Prentice-Hall International, Inc.

Godfrey, K. (1993). Perturbation Signals for System Identification. Prentice Hall.

Nelles, Oliver (2001). Nonlinear System Identification. Springer.

Popović, D. and T. Sinkjær (2000). Control of Movement for the Physically Disabled. Springer. 